

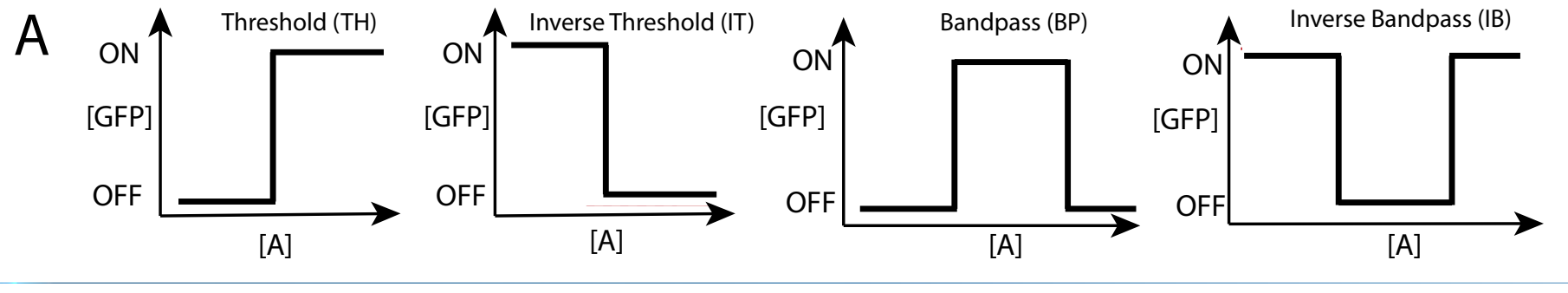

B
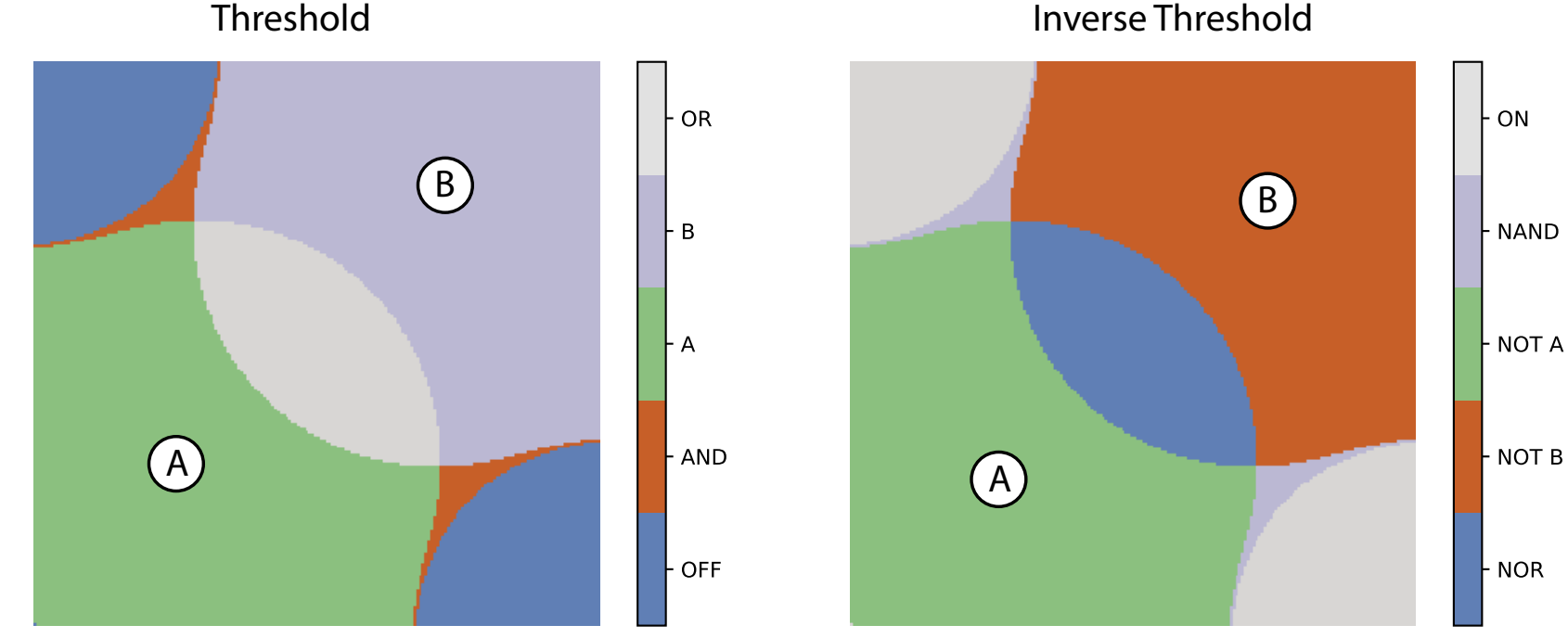

Bandpass
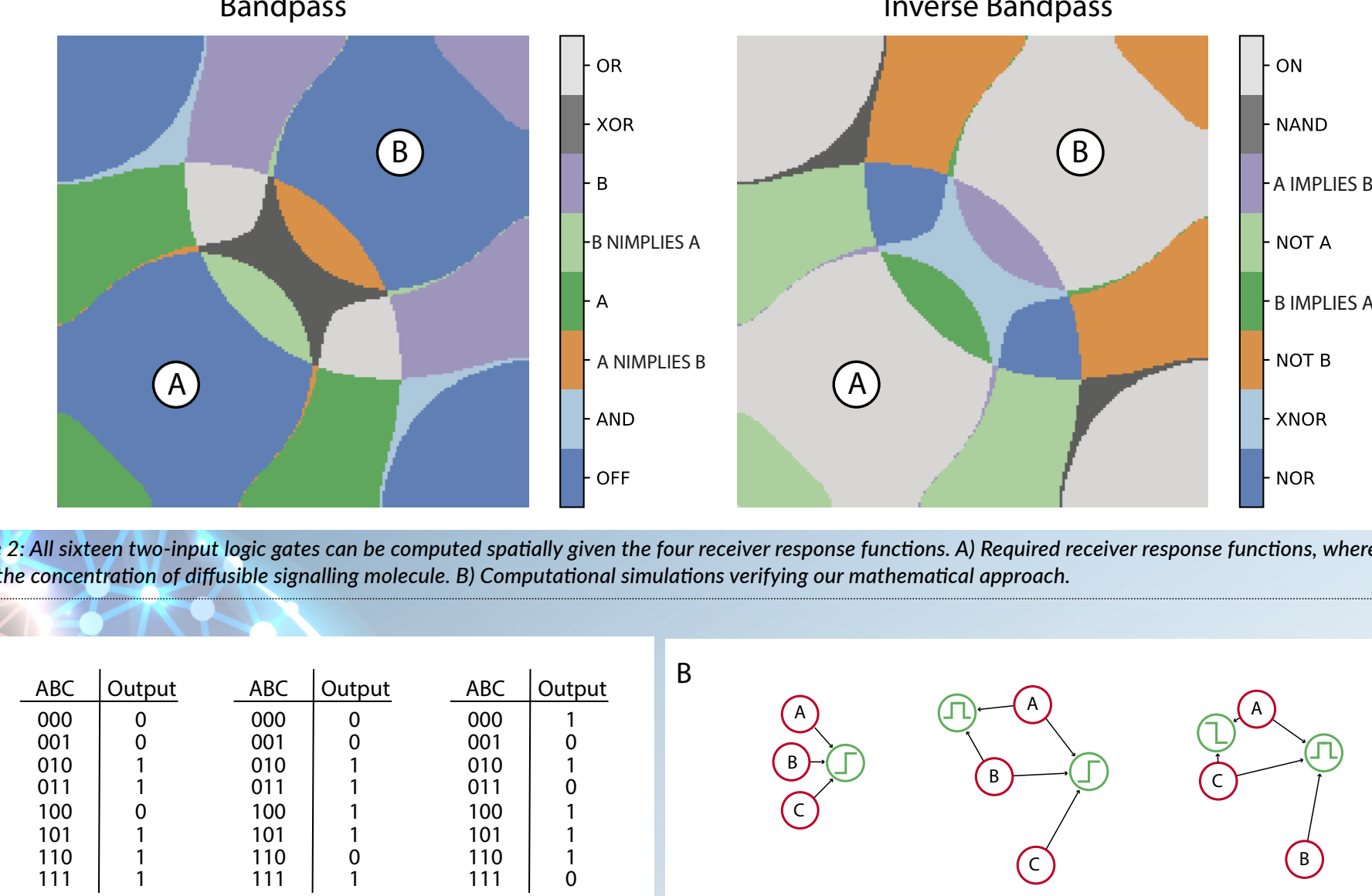
(A)
()

$\overbrace{B}^{-1}-()$

(c)
Figure 2: All sixteen two-input logic gates can be computed spatially given the four receiver response functions. A) Required receiver response functions, wher order of increasing signal concentration. The relative concentrations of the input states can change depending on the position of the receiver colony. For input $A$, the orderwould be $00,01,10,11$ at the receiver for 10 would be higher at the receiver for 10 would be higher than 01 . Similarly, if the output colony be $00,10,01,11$.

Furthermore, we represent the activation functions as boundaries partitioning the input states into $\mathrm{ON}$ an OFF boxes. For example, the threshold partitioning would be OFF|ON where mapped to OFF, and anything to the right is mapped to ON. This represents the activation pattern of the threshold, which is inactive at low signalling molecule concentrations and active high concentrations. Therefore, the AND gate in Figure 1 would be represented as $00,01,10 \mid 11$, where the 11 input state is alone on the right of the boundary as it is the only one in which the output colony is activated.

Using this representation, we can enumerate all the distinct logic gates that are possible given the properties that are system. We used this roperties to show that all possible two-input logic functions cas be computed spatially, provided we have receivers with response functions given in Figure 2A. We verifed this with computer simulations (Figure 2B). This is effectively a map of the area around the senders, where the colour of each would be encoded if a receiver was placed in that region. We can clearly see that the function encoded by given receiver is dependent on its position relative to the inputs and that between the four activation function we can encode all 16 two input logic gates as predicted.

We then went on to show that we can construct any Boolean function by using two layers of digital logic (effectively multiple different receiver colonies) example, if the receiver is very close to anything to the left of the boundary is logic functions cas be two-input region represents the logic gate tha

In this case, the function output is if any receiver colony fluoresces. This demonstrates the strength of the approach and proves that arbitrary spatial computation is possible. A key consideration when building electronic digital circuits is electronic design automation (EDA). EDA comprises a category of algorithms that find the simplest implementation of a given function using the available electronic parts. We have developed an analogous design algorithm that given a specified digital function, finds the simplest spatial pattern that implements the function using the smallest number of bacterial colonies. Figure 3 shows simplified designs for three different Boolean functions.

\section{Future directions}

- We are currently engineering $E$. coli strains that function as senders and receivers. We will verify our mathematical approach experimentally and generate configurations that can compute arbitrary digital functions.

- The approach to building arbitrary analogue functions will be extended.

- We will investigate more complex computing based on cellular automata (CA). These are discrete dynamical systems comprising 'cells' on a grid that update their state based on the states of their neighbours. These simple local rules are well known to generate complex behaviours but can also perform computation through changing spatial patterns and can be equivalent to Turing machines and neural networks. This will allow different computations, examples of which are temporal logic (which signals arrived first), counting of events, and supervised learning (distinguishing between sets of inputs). This architecture will enable the next generation of computational biosensor devices.
PROJECT NAME

SynBioBrain: Buildling biological

PROJECT SUMMARY

using synthetic biology, we can now engineer bacteria into whole-cel biosensors where sensing, transduction
and output Applications include the detection harmful environmental agents, bioprocess relevant bismarkers Engineering microb consortia allows biosensor informatic to be integrated and processed in
distributed fashion. In this project, construct biological computers formed communicate using intercellular signalling ways to p. Our approach opens up

PROJECT LEAD

Professor Barnes originally received a PhD in particle physics then became interested n biological systems. He joined UCL's Faculty of Life Sciences in 2012. Chris's and he has built an outstanding funding and publication record. His research is multidisciplinarity, combining mathematical machine learning and molecular biology.

CONTACT DETAILS

Professor Chris Barnes

519 Rockefeller Building 21 University Street

M christopher.barnes@ucl.ac.uk

(1) https://ucl-cssb.github.io/

(2) @cssb_lab erc

FUNDING

Tinis project has received funding from the European
Research Council (ERC) under the European Union's Horizon 2020 research and innovation programme Jiant agreement No. 770335 . 